\title{
Research on Digital Library Based on Virtual Reality Technology
}

\author{
Cuiyuan Zhang \\ Mudanjiang Normal University, Mudanjiang City, Heilongjiang Province 157012, China \\ zcycola@sina.com
}

Keywords: Virtual reality; digital library; 3D modelling; visualization; interaction.

\begin{abstract}
To explore intelligent library, the method of literature survey, case analysis, empirical research method, comparative analysis method, interdisciplinary research and other research methods were adopted to make an all-round discussion of application foundation of the digital library, system composition and realization and necessity and feasibility of technology in the digital library. In addition, the application of digital technology in the digital library virtual space, 3D information resources construction, virtualization information retrieval, virtual reference consulting and remote control technology was analysed. The necessity of applying technology in digital library was illustrated from the perspective of development of information society, digital library construction and development and innovative services. Starting from the principle and characteristics of the virtual reality modelling language, through the virtual space design of digital library, the application of virtual reality technology in digital library virtual space was explored from the system four types. The problems existing in applying virtual reality technology in the digital library were analysed. The results showed that pure software technology was the best choice for China's digital library at present and distributed system combining pure software technology and virtual reality technology was the development direction of the application of digital library. To sum up, the two dimensional information resources and three dimensional information resources are compared and analysed by using the concrete realization of the information resources construction.
\end{abstract}

\section{Introduction}

As an important part of the infrastructure of the information age, digital libraries have been paid more and more attention to at home and abroad. The rapid development of the digital library not only brings a broad development opportunity to the development of the traditional library, but also puts forward a series of severe challenges. As a distributed, spatial-temporal, convenient and large-scale information centre, digital library has a brand-new way of organization and management. It has become the trend of library construction and development nowadays [1]. Since the middle of 1990s, the research and development of digital libraries began in China. The implementation of the "China Experimental Digital Library" project approved by the State Planning Commission in 1997 marked the start of the construction of digital library in China.

Virtual reality technology is considered to be one of the most important and new research directions in the field of computer in the 21st century. People pay much attention to the three key technologies, virtual reality technology, Internet and multimedia technology. Virtual reality is a comprehensive technology integrating multi-disciplinary and multi-fields. It involves computer science, network technology, distributed technology, robot technology and so on [2]. The most characteristic is that $3 \mathrm{D}$ modelling function of the virtual reality can construct vivid virtual environment in the computer. People can, through the use of some sensing devices, very conveniently, intuitively and naturally "enter" the virtual space, immerse in it and have interactive reverie. The achievement of virtual reality should first of all create a virtual world in the computer environment, and the virtual world can produce perception role of different organs including visual, auditory and tactile. Secondly, the experiencer can naturally interact with the virtual world created by computer through the basic behaviours and actions of person. Thirdly, virtual reality can make use of digital gloves, 3D interactive ball and head anti-theft display sensor and display device to enhance interactivity and improve the real sense of experiencer [3]. In the past more than 20 years, the scope 
of virtual reality applications has been expanding. In foreign countries, it has developed from the earliest applications of military, space and other advanced fields to ordinary folk. In China, the state attaches great importance to the research and application of virtual reality. It not only includes virtual reality in major research projects, but also strongly supports the application and popularization of virtual reality.

\section{Application Foundation of Virtual Reality Technology in Digital Library}

The 3D modelling function of VR can create a virtual world of multiple perception such as visual, listening, sense and touch. The virtual world can be completely fictional and it can also be virtual reproduction of the reality world, namely the environment of "reality in virtualization and virtualization in reality" in virtual reality. As a result, people immersed in the sense of vision, hearing, touch, smell and so on produced in this virtual environment. VR's demand for immersion and mutual inductance requires real-time computer processing ability. The natural and harmonious human-machine interaction environment can increase immersion to experiencer and change the rigid, boring and passive state between human and computer [4]. Although there is no clear definition at present, there are three essential systems for the generation and implementation of the narrow sense system from the point of view of the technical realization, as shown in Figure 1.

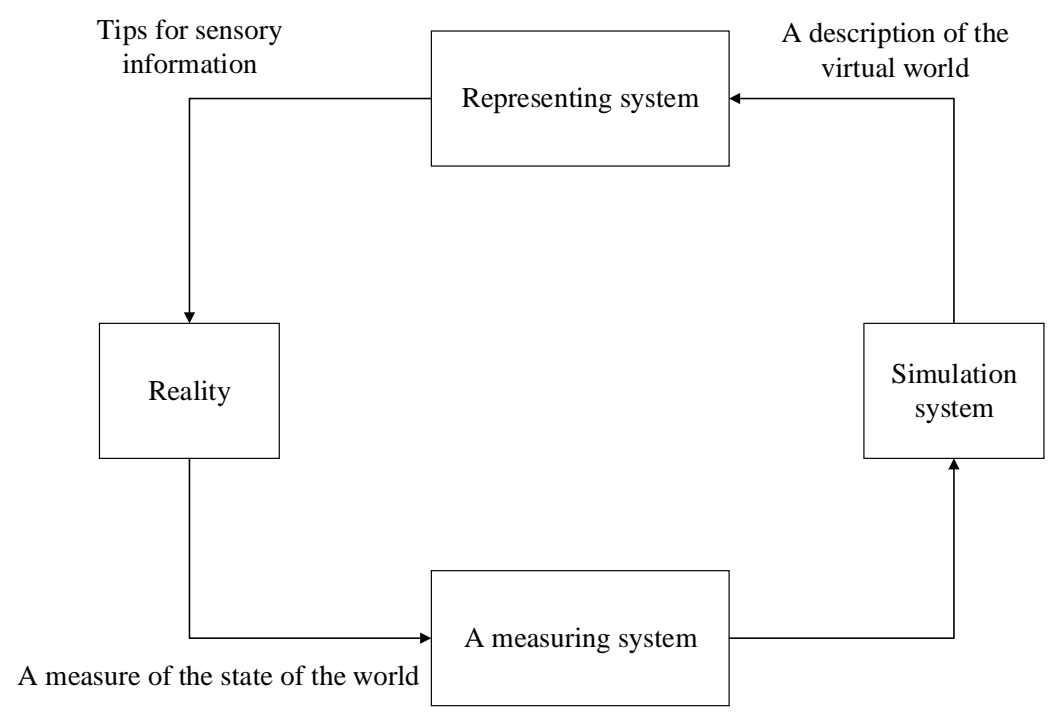

Figure. 1 Realization of the structure of virtual reality

\section{Application of Virtual Reality Technology in Digital Library}

\subsection{Realization of 3D Information Resources of Digital Library Based on Virtual Reality Technology}

As the mainstream three-dimensional modelling method, virtual reality technology has very strong modelling and simulation ability so that it can be very convenient to realize the construction of three-dimensional information resources. The traditional information processing environment has always been "people adapt to computers". When virtual reality technology came into being, people began to have the concept of "computer adapts to humans", and people became the master of information processing environment, which is a brand new idea. People can participate in the information processing environment through vision, hearing, touch, smell and so on, as well as form, gesture or password, so as to get "real" experience. The system is no longer built on a single dimensional digital space, but built in a multidimensional information space. Virtual reality technology is the key technology to support this multidimensional information space [5].

The visualization of three-dimensional information introduces the concept of volume so that it transcends two-dimensional information. The visualization of three-dimensional information is widely used in scientific computing. In the resource construction of digital library, some digital 
libraries have made use of virtual reality technology to carry out the construction and service of three-dimensional information resources. In foreign countries, the British Library has first carried out the construction and service of the three-dimensional books, mainly using the display equipment to provide the valuable literature of the library collection. At home, the Chinese national library has made a deliberate attempt in this respect.

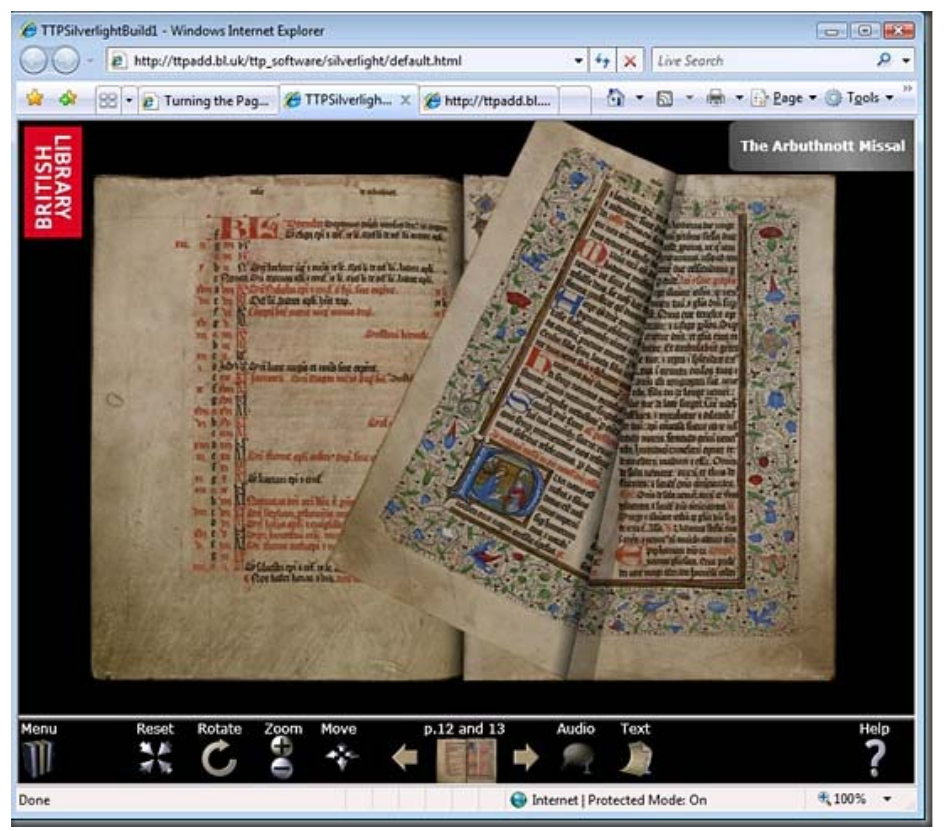

Figure. 2 Three-dimensional virtual reading of the British Library

\subsection{Application of Virtual Reality Technology in the Information Retrieval of Digital Library}

Information retrieval visualization belongs to information visualization. Information retrieval visualization refers to converting the data and its semantic relations in data set into visual display, and displaying the internal retrieval process to users. Information retrieval visualization generally includes two aspects including visualized information display and visualized information retrieval. It is mainly the visualization of information retrieval process and the visualization of retrieval results, in which the visualization of retrieval results is the most important. Computer information retrieval is closely related to the way of information organization. From the perspective of information retrieval, it can be said that the way of information organization determines the way of information retrieval [6].

The visualized cross library retrieval system text mode and graphical mode of Chinese National Library of Science can realize theme visualization, database visualization, age visualization and author visualization. Figure 1 is a database visualization window, with a pie chart revealing the retrieval architecture. The "virtual reality technology" retrieval uses database including China Journal Full-text Database, VIP, Vepsa Chinese Scientific and Economic News Database, Chinese Doctoral Dissertation Database and Chinese Outstanding Master's Degree Thesis Database; Figure 2 is "the year issued amount of papers for "virtual reality technology". Users can not only interact with the system in the graphical interface, but also reveal the hidden rules and associations in the retrieval results from multiple angles. 


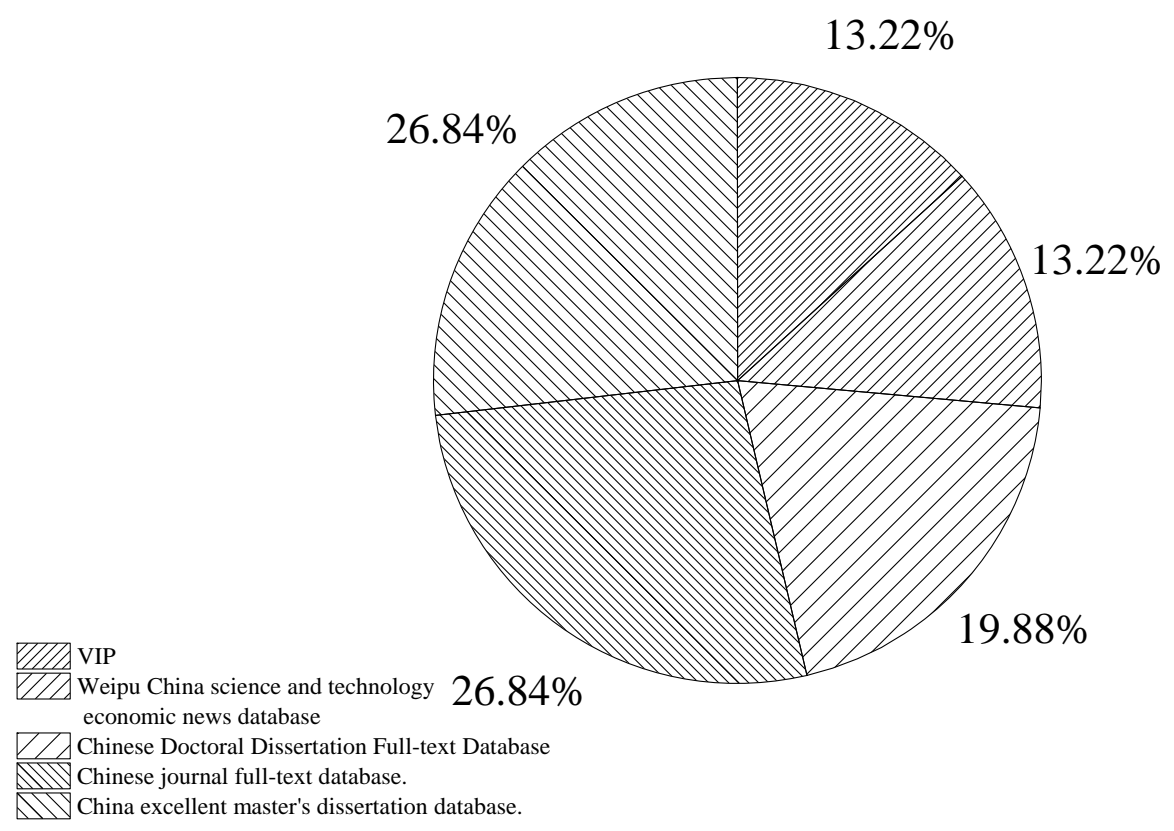

Figure. 3 Virtual reality technology database visualization

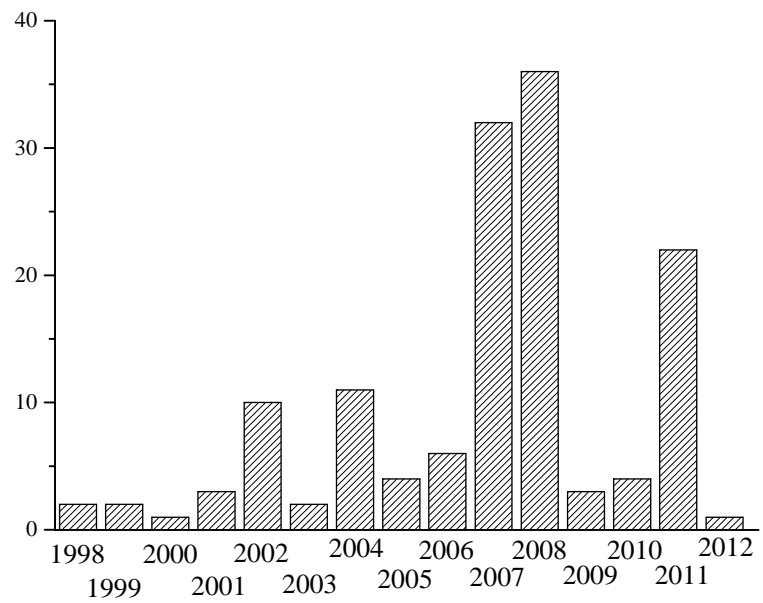

Figure. 4 Virtual reality technology age visualization

The user is visually interacting with the computer from virtualization, thus making a new service model applied to the digital library. In addition to the physical library digital library in carrying out the research and application of visual information retrieval, digital library service based on the database also joined the application of information retrieval visualization. For example, Wanfang Data knowledge service platform in information retrieval also increased the knowledge context analysis, including comparative analysis and retrieval of knowledge context. 


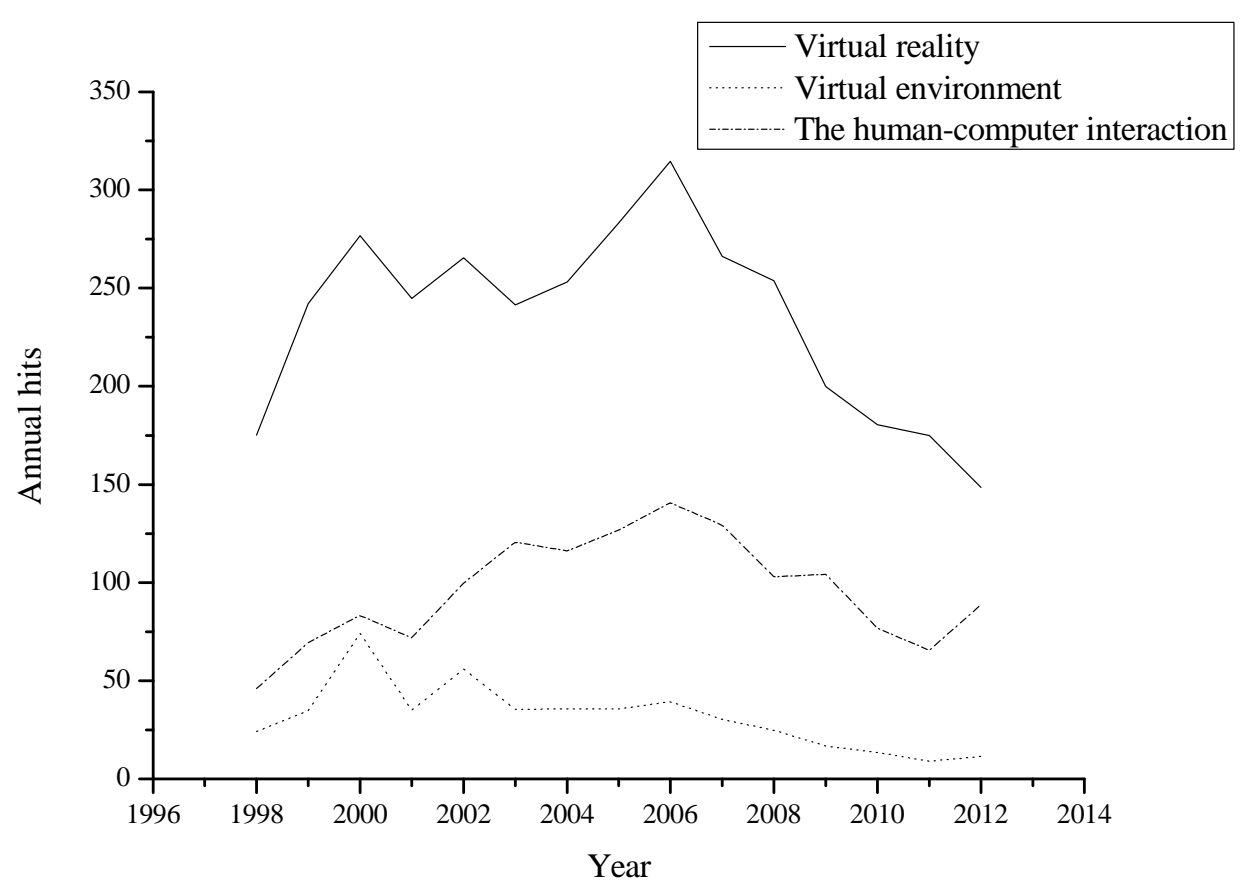

Figure. 5 Comparison and analysis of the Wanfang data knowledge service platform

\subsection{Application of Virtual Reality Technology in Digital Library Reference Consulting}

The essence of reference consulting is the interaction between reference consulting librarians and users to help users solve problems in the process of using the library. Virtual reality technology combines multimedia technology, visualization technology, network technology, artificial intelligence technology and distributed processing technology. It has the functions of natural interaction, perception immersion, experience imagination and it is applied in digital library reference service. It integrates the application of comprehensive innovative technology and intelligent and advanced technology, which will promote digital library service innovation [7].

The application of virtual reality technology in the reference service of digital library is limited by the hardware and software and network conditions. At the present stage, the virtual interactive reference service and the application of virtual space conference system can be carried out. In the aspect of visual reference of the digital library, currently, part of the library begins to try visualization digital reference consulting. For instance, real-time video reference consulting of the Ohio University library not only provides AOL Instant Messenger, Yahoo! Messenger and MSN Messenger three instant messaging reference consulting ways, but also uses the free phone system, which achieves the "Skype a Librarian" and conducts the consulting questions and answers from video instant messaging. In iTunes Arizona State University library, we launched the video podcast series, including 3-5 minutes of video for search and how to use library to do research.

\section{Conclusion}

The digital library is the extension of the traditional library in the information age. It not only includes the functions of the traditional library, but also integrates some functions of other information resources institutions, such as museums, archives, publishing houses, etc., which can provide comprehensive public information access service. The digital library is not simply uploading the information resources of the traditional library to the Internet, but an object oriented, distributed collection of digitalized resources with network access function.

Virtual reality technology is a comprehensive technology integrating the field of computer graphics, human-computer interaction technology, sensor technology, artificial intelligence, robotics and other disciplines. It uses computer to generate 3D visual and auditory multiple feeling, make participants naturally experience and interact with the virtual world through appropriate devices. 
The interdisciplinary and multidisciplinary integrated technology plays a very important role in the digital library construction. The construction of the digital library needs to use computer technology, network technology and many other technologies, including digital technology, multimedia technology, information organization, compression, storage, transmission, retrieval, analysis technology, large scale database technology security technology and so on. Therefore, the three-dimensional modelling, multi-perception, immersion, interaction and imagination of virtual reality technology can be fully reflected in the application of digital library.

\section{Acknowledgments}

This paper is the scientific research project of the Work Committee of the University Library of Heilongjiang province (2017-A-094).

\section{References}

[1]. Bastug E, Bennis M, Medard M, et al. Toward Interconnected Virtual Reality: Opportunities, Challenges, and Enablers. IEEE Communications Magazine, 2017, 55(6), pp, 110-117.

[2]. Faisal A. Computer science: Visionary of virtual reality. Nature, 2017, 551(7680), pp, 298-299.

[3]. Freeman D, Reeve S, Robinson A, et al. Virtual reality in the assessment, understanding, and treatment of mental health disorders. Psychological Medicine, 2017, 47(14), pp, 2393.

[4]. Standen P J, Threapleton K, Richardson A, et al. A low cost virtual reality system for home based rehabilitation of the arm following stroke: a randomised controlled feasibility trial. Clinical Rehabilitation, 2017, 31(3), pp, 340.

[5]. Limanowski J, Kirilina E, Blankenburg F. Neuronal correlates of continuous manual tracking under varying visual movement feedback in a virtual reality environment. Neuroimage, 2017, 146, pp, 81-89.

[6]. Thomsen A S, Bachholm D, Kjærbo H, et al. Operating Room Performance Improves after Proficiency-Based Virtual Reality Cataract Surgery Training.. Ophthalmology, 2017, 124(4), pp, 524-531.

[7]. Gou F, Chen H, Li M C, et al. Submillisecond-response liquid crystal for high-resolution virtual reality displays. Optics Express, 2017, 25(7), pp, 7984. 